\title{
ANÁLISE DO PROCESSO DE MUDANÇA ESTRATÉGICA SOB A PERSPECTIVA DA TEORIA DAS CAPACIDADES DINÂMICAS: 0 caso da metalúrgica inovação
}

\author{
Gabriela CAPPELLARI $^{1}$ \\ Janice WALTER ${ }^{2}$ \\ Jorge Oneide SAUSEN ${ }^{3}$
}

\author{
${ }^{1}$ Mestranda, Unijuí, gabriela.cplr@gmail.com \\ ${ }^{2}$ Mestranda, Unijuí, janice@sommacontabilrs.com.br \\ ${ }^{3}$ Doutor, Unijuí, josausen@unijui.edu.br
}

Recebido em: 13/04/2016 - Aprovado em: 20/11/2017 - Disponibilizado em: 30/12/2017

\begin{abstract}
RESUMO
O estudo descreve o processo de mudança estratégica da Metalúrgica Inovação, localizada na cidade de Santa Rosa, região Noroeste do Estado do Rio Grande do Sul, no período de 2000 a 2015. A pesquisa caracteriza-se como qualitativa, exploratória, descritiva e estudo de caso. Os dados foram analisados com base na direct research e interpretados com base na teoria das capacidades dinâmicas. A análise revelou que as mudanças ocorridas na Metalúrgica Inovação foram conduzidas ora pela capacidade de identificar oportunidades do empresário, ora pelo ambiente. As decorrências vão ao encontro da teoria das capacidades dinâmicas e de seus fatores componentes, sendo a capacidade absortiva, adaptativa e inovativa. Esta teoria apresentou-se adequada para explicar o contexto da organização, uma vez que trata-se de uma abordagem contemporânea que melhor elucida o enredo da empresa.

Palavras-chave: Mudança. Capacidades Dinâmicas. Vantagem Competitiva.
\end{abstract}

\begin{abstract}
The study describes the strategic change process of Metallurgical Innovation, located in Santa Rosa, the State of Rio Grande Northwest South, from 2000 to 2015. The research is characterized as qualitative, exploratory, descriptive and study affair. Data were analyzed based on direct research and interpreted on the theory of dynamic capabilities. The analysis revealed that the changes in Metallurgical Innovation were conducted prays for the ability to identify the entrepreneur, now the environment. The moves are in line with the theory of dynamic capabilities and its component factors, and the absorptive capacity, adaptive and innovative. This theory showed to be adequate to explain the context of the organization, since it is a contemporary approach that best explains the plot of the company.
\end{abstract}

Keywords: Change. Dynamic Capabilities. Competitive Advantage.

\section{INTRODUÇÃO}

O ambiente mercadológico apresentase cada vez mais incerto, complexo e altamente competitivo. Assim, diante de um contexto de descontinuidade as mudanças são tão grandes e rápidas que se torna difícil, por vezes, compreender a relação de causa e efeito dentre elas. Nesta perspectiva, Sminia (2009) orienta que as organizações necessitam encontrar alternativas inovadoras e criativas para que seja possível a construção de processos de melhoria contínua, contemplando também ações para a mudança organizacional.

Destarte, a adaptação estratégica é essencial à sobrevivência organizacional, sobretudo pelo descomedido grau de inquietação, complexidade e competitividade que o ambiente tem-se apresentado nos últimos tempos (SAUSEN, 2012). Uma vez que adaptação é contínua, as empresas garantem vantagens competitivas cada vez 
mais agressivas e sustentáveis formando um conhecimento em espiral (WHITTINGTON, 2002).

Mintzberg, Ahlstrand e Lampel (2010) reiteram que é primordial observar o caráter descontínuo e mutável do ambiente na análise das mudanças organizacionais. Pettigrew (1987) salienta que apesar da pressão do ambiente, as empresas podem decidir quanto a suas estratégias, ainda que sejam limitadas de alguma forma pelo cenário.

Diante do exposto, o objetivo deste estudo foi analisar o processo de mudança estratégica sob a perspectiva da teoria das capacidades dinâmicas na empresa Metalúrgica Inovação Ltda, instalada na cidade de Santa Rosa, região Noroeste do Estado do Rio Grande de Sul.

A pesquisa justifica-se pela relevância. A análise do processo de mudança e a adaptação de acordo com a teoria das capacidades dinâmicas é fundamental, pois, explica como a empresa trabalha com as adversidades e se empenha pela sua sobrevivência.

O estudo está estruturado em quatro partes, além da introdução. Na primeira apresenta-se a revisão bibliográfica que discorre sobre capacidades dinâmicas e mudança organizacional e adaptação estratégica. $\mathrm{Na}$ segunda parte são apresentados os procedimentos metodológicos utilizados na investigação empírica. Logo após a apresentação e análise dos resultados e por fim, as considerações finais do estudo.

\section{REVISÃO BIBLIOGRÁFICA}

\subsection{Capacidades Dinâmicas}

O estudo das capacidades dinâmicas compõe um campo de atividades de pesquisas em gerenciamento estratégico, mudanças organizacionais e vantagem competitiva (CAMARGO; MEIRELLES, 2012). O conceito é relevante porque se trata da capacidade adaptativa da organização frente ao dinamismo do ambiente, ou melhor, a forma pela qual as empresas podem alcançar e sustentar vantagens competitivas em um ambiente mutável (NELSON, 1991; TEECE; PISANO; SHUEN, 1997; DOSI; NELSON; WINTER, 2000; TEECE, 2009).

Deste modo, as capacidades dinâmicas são uma corrente explicativa de vantagem competitiva avançada e contemporânea que explica o momento em que as organizações estão passando, considerando ambientes em constante transformação. Mediante a reconfiguração da estrutura, processos, rotinas e recursos as empresas buscam atender as exigências do mercado intensamente dinâmico.

Winter (1964) foi o pioneiro nos estudos das capacidades dinâmicas e desde a sua proposta original, vários são os esforços teóricos para desenvolver o conceito, especialmente do ponto de vista dos microfundamentos e operacionalização (ZOLLO; WINTER, 2002; WANG; AHMED, 2007; TEECE, 2009).

Alguns autores focam suas análises nos aspectos internos da organização e para 
estes, a existência das capacidades dinâmicas esta relacionada com processos estratégicos e operacionais (NELSON; WINTER, 1982), ou então com a habilidade da empresa em desenvolver novas estratégias através do reconhecimento de recursos de valor frente a concorrência (COLLIS, 1994). Em contrapartida, outros pesquisadores relacionam as capacidades dinâmicas com o dinamismo do ambiente, isto é, ambientes com mudanças intensas e constantes (TEECE; PISANO; SCHUEN, 1997; WANG; AHMED, 2007).

Existem outros estudiosos que investigam os mecanismos e dispositivos que configuram a existência de capacidades dinâmicas (ZOLLO; WINTER, 2002). São autores que destacam o uso de mecanismos automáticos de mudança (WINTER, 2003). Nesta linha de pensamento, Zollo e Winter (2002) defendem que as organizações são capazes de integrar, construir e reconfigurar suas competências mesmo em ambientes pouco dinâmicos e com taxas de mudanças reduzidas. Destarte, as capacidades dinâmicas são fundamentadas na existência de mecanismos rotineiros que permitem reconfigurar as competências das empresas (WINTER, 2003; ANDREEVA; CHAIKA, 2006).

Ainda outros autores associam as capacidades dinâmicas ao resultado de uma combinação destas, isto é, que são definidas com base em uma hierarquia de capacidades simples e rotinas relacionadas
(EISENHARDT; $\quad$ MARTIN, 2000; ANDREEVA; CHAIKA， 2006; WANG; AHMED, 2007).

Wang e Ahmed (2007) definem as capacidades dinâmicas como a orientação comportamental de uma organização constantemente para integrar, reconfigurar, renovar e recriar seus recursos e, mais importante, atualizar e reconstruir as suas competências em resposta a mudanças no ambiente para alcançar e sustentar uma vantagem competitiva. $\mathrm{Na}$ ótica destes autores, as capacidades dinâmicas não são somente processos, mas algo embutido nos processos. Os autores explicam que processos normais não possuem relação e que este tipo de processo pode ser codificado. Então, por consequência são vulneráveis de serem transferidos com facilidade entre organizações, por isso, não constituem elementos de vantagem competitiva sustentável. Wang e Ahmed (2007) apresentam ainda três elementos componentes das capacidades dinâmicas: a capacidade adaptativa, a capacidade absortiva e a capacidade de inovação.

*Capacidade absortiva: habilidade da empresa em adquirir conhecimento externo, assimilá-lo com o interno, criando assim mecanismos para explorar este novo saber.

*Capacidade adaptativa: habilidade da empresa em identificar e capitalizar as oportunidades emergentes do mercado. Reforça também a habilidade da empresa em se adaptar no tempo certo por meio de 
flexibilidade dos recursos e alinhamento de seus recursos e capacidades com as mudanças do ambiente.

*Capacidade de inovação: habilidade da empresa em desenvolver novos produtos e mercado, através da orientação do alinhamento estratégico para comportamentos e processos de inovação.

Apesar do destaque feito pelos autores às capacidades como fatores componentes das capacidades dinâmicas, em seu modelo de análise é proposto também processos adjacentes, que envolvem a integração, reconfiguração, renovação e recriação dos processos específicos da empresa.

Portanto, o modelo das capacidades dinâmicas explica como as empresas podem agir para reconfigurar a sua base de recursos, mesmo operando diante de incertezas e instabilidades do contexto concorrencial (VASCONCELOS; CYRINO, 2000). Esta literatura enfatiza a necessidade das empresas de desenvolver competências de forma a criar vantagem competitiva como estratégia primordial para a superação dos concorrentes (SAUSEN, 2012).

\subsection{Mudança organizacional e adaptação estratégica}

As mudanças organizacionais são incorporadas na vida das organizações de modo natural. Pettigrew (1987) defende que para compreender as mudanças faz-se necessário examinar a justaposição do analítico e do político no desempenho das pessoas em situações de crises, quando suas habilidades são testadas pelas forças da interação com o ambiente. Assim, entende-se que a mudança não é um evento isolado, mas um processo que envolve a percepção dos agentes envolvidos, avaliação das condições do ambiente e a escolha de estratégias.

Cohen (1999) realça que as mudanças ocorrem em todos os domínios da empresa, como em sistemas de gestão da informação, estratégias de negócios, propriedade e estruturas organizacionais e relacionadas à tecnologia. Neste sentido, as organizações são reconhecidas como sistemas políticos e culturais, onde as mudanças podem ser analisadas em seu contexto, conteúdo e processo e as suas interligações (PETTIGREW, 1992). Neste sentido, a mudança é vista como uma consequência e não apenas como solução de problemas. $\mathrm{Na}$ ótica de Pettigrew (1992), a mudança organizacional é produto de um processo que reconhece a luta histórica e contínua da empresa.

Child e Smith (1987) asseveram que cada setor da empresa é uma porta de entrada para a mudança e as mudanças podem ser investigadas em seu contexto, conteúdo e processo (PETTIGREW, 1987). No contexto evidenciam-se as condições antecedentes à mudança, que está interligada com a estrutura interna e externa da empresa (é o "por que" da mudança). O conteúdo relaciona-se com as áreas de transformação, como mudar a tecnologia, a força de trabalho ou a própria 
cultura organizacional (é o "o que" da mudança). Por fim, o processo considera as ações, reações e as interações da empresa do seu estado presente para o futuro (é o "como" das mudanças). Destarte, o processo de mudança organizacional possui caráter de processo político de aprendizagem a longo prazo.

Nesta perspectiva, Hrebiniak e Joyce (1985) apresentam um modelo de adaptação organizacional. Este modelo propõe que as escolhas e as capacidades das empresas são independentes e que é possível esclarecer o comportamento gerencial por meio destas variáveis. Os autores asseguram que a adaptação é um processo dinâmico sendo resultado de força relativa e tipo de poder ou dependência entre organização e ambiente. Por fim, destacam ainda que a escolha estratégica e o determinismo ambiental fornecem impulso para o processo de mudança, cada um é insuficiente e ambos são necessários para a explicação satisfatória da adaptação organizacional.

No quadrante I é demonstrado que as escolhas são possíveis, porém limitadas devido à falta de recursos e força da organização face a face com o ambiente. No quadrante II, a escolha estratégica é alta, mas seletiva ou diferenciada por causa do alto poder compensatório e excesso de recursos existentes no ambiente. Já no quadrante III, as dependências por recursos da organização são menores e o número de opções estratégicas são maiores. Por fim, quanto ao quadrante IV, as escolhas organizacionais são incrementais e a adaptação acontece por acaso, uma vez que são baixa a escolha estratégica e o determinismo ambiental.

\section{METODOLOGIA}

A pesquisa foi realizada na Metalúrgica Inovação, localizada na cidade de Santa Rosa, região Noroeste do Estado do Rio Grande do Sul. O estudo caracteriza-se como qualitativo (MINAYO, 2008), exploratório (ANDRADE, 1999; MARTINS, 2000), descritivo (TRIVIÑOS, 1987; PRODANOV; FREITAS, 2013; GERHARDT; SILVEIRA, 2009) e estudo de caso (YIN, 2005; CRESWELL, 2010).

A coleta de dados foi realizada por meio de entrevista semiestruturada com o proprietário da Metalúrgica Inovação, pois esta técnica valoriza a presença do investigador e oferece liberdade e espontaneidade ao informante, enriquecendo assim a investigação (YIN, 2005). Foram realizadas duas entrevistas com o empresário, tendo duração de cerca de três horas cada, identificando os episódios que marcaram a organização no período do ano 2000, quando a mesma foi fundada, até o ano de 2015. Tais acontecimentos foram organizados na linha do tempo e identificados em três períodos: (a) concepção do modelo de negócio; (b) crescimento e expansão do mercado; (c) crise e fusão.

Para nortear a coleta e a análise dos dados da presente pesquisa, foram adotados 
os procedimentos propostos pela direct research, idealizada por Mintzberg (1978). A direct research utiliza a análise longitudinal, evidenciando a evolução das estratégias num período histórico determinado, esclarecendo ao pesquisador o processo de mudança e adaptação estratégica.

Pettigrew, Ferlie e McKee (1992) ainda consideram três elementos fundamentais para o entendimento do processo evolutivo e de mudança organizacional: o conteúdo da mudança (o que); o contexto da mudança (por que); e o processo da mudança (como). O conteúdo relaciona-se às ações que dão consistência a uma decisão. $\mathrm{O}$ contexto serve de justificativa à decisão. O processo, contudo, é a forma pela qual a decisão foi implementada. Estes três elementos foram considerados na descrição e análise do caso estudado.

\section{ANÁLISE E INTERPRETAÇÃO DOS RESULTADO}

\subsection{Períodos de Mudança Estratégica na} Empresa

A seguir são apresentados os eventos marcantes dentre os anos 2000 a 2015, de acordo com a metodologia do estudo, interpretadas à luz da teoria das capacidades dinâmicas. A Figura 1 apresenta a sistematização dos períodos estratégicos que determinaram as principais mudanças na Metalúrgica Inovação.

Figura 1-Períodos de mudança estratégica na Metalúrgica Inovação

\begin{tabular}{|c|c|c|c|c|}
\hline & Períodos Estratégicos & $\begin{array}{l}\text { Contexto } \\
\text { (Por que?) }\end{array}$ & $\begin{array}{l}\text { Conteúdo } \\
\text { (O que?) }\end{array}$ & $\begin{array}{l}\text { Processo } \\
\text { (Como?) }\end{array}$ \\
\hline $\begin{array}{l}2000 \\
2005\end{array}$ & $\begin{array}{l}\text { I - Concepção do } \\
\text { modelo de negócio }\end{array}$ & $\begin{array}{c}\text { Visão de } \\
\text { oportunidade }\end{array}$ & $\begin{array}{l}\text { Preparação da empresa para } \\
\text { ser fornecedor e adequações } \\
\text { aos diferentes clientes }\end{array}$ & Processos de melhoria \\
\hline $\begin{array}{r}2006 \\
\text { a } \\
2010\end{array}$ & $\begin{array}{l}\text { II - Crescimento } \\
\text { e expansão do } \\
\text { mercado }\end{array}$ & $\begin{array}{c}\text { Abertura de novos } \\
\text { clientes }\end{array}$ & $\begin{array}{l}\text { Desenvolvimento de produtos } \\
\text { de acordo com a necessidade } \\
\text { específica de cada cliente }\end{array}$ & $\begin{array}{c}\text { Desenvolvimento } \\
\text { concomitante, necessidade } \\
\text { contínua de adaptação }\end{array}$ \\
\hline $\begin{array}{c}2011 \\
\text { a } \\
2015\end{array}$ & III - Crise e fusão & $\begin{array}{l}\text { Crise política com } \\
\text { reflexos } \\
\text { econômicos }\end{array}$ & $\begin{array}{c}\text { Fusão da Metalúrgica com a } \\
\text { empresa Stara }\end{array}$ & $\begin{array}{l}\text { Processo de mudança da } \\
\text { organização e adaptação }\end{array}$ \\
\hline
\end{tabular}

Fonte: Dados da pesquisa (2015)

\subsubsection{Período I - Concepção do modelo de negócio (2000 - 2005)}

A Metalúrgica Inovação está em funcionamento a cerca de 15 anos e a ideia da abertura do negócio partiu da visão de oportunidade do proprietário, considerando a alavancagem da mecanização do segmento agrícola na época. Na oportunidade a empresa por meio de processos de melhoria, adaptou- 
se para atender diferentes clientes como fornecedor.

Em entrevista, o empresário explica que a principal forma de captura de conhecimento e informações atualizadas do segmento e concorrentes foi a participação em feiras nacionais e internacionais e estreito relacionamento com os clientes atuais $\mathrm{e}$ potenciais. De forma contínua e abrangente a empresa acompanhava as tendências, buscando proativamente explorar novas oportunidades e atender as específicas necessidades da clientela.

Através da abertura para estágios e estudos de conclusão de curso de programas de graduação e SENAI, buscava novos conhecimentos para aperfeiçoar e inovar a gestão como também o processo produtivo. Neste período, o desenvolvimento de novas tecnologias e maneiras diferentes de produção com as inovações presentes no mercado úteis à organização eram analisadas informalmente, conforme a necessidade da empresa e de seus clientes.

Em relação às dificuldades encontradas neste evento, destaca-se a resistência à mudança por parte da equipe de trabalho, composta por aproximadamente 40 trabalhadores.

Os treinamentos, a gestão e a hierarquia da empresa foram fatores-chave no benchmarking industrial e na gestão do conhecimento, oferecendo à organização suporte na absorção e adaptação das rotinas, tecnologias e processos bem sucedidos.
A ênfase era surpreender e satisfazer a clientela e para isto a empresa investigava o perfil de cada cliente em específico. Com isso era possível identificar e/ou eliminar conhecimento e práticas internas possivelmente obsoletas para determinado processo produtivo, renovando desta forma as competências, estimulando e adaptando-se à busca por inovações e melhorias alternativas.

O aproveitamento do conhecimento era $o$ foco principal da equipe de desenvolvimento de produtos. $\mathrm{O}$ cliente recebia suporte e acompanhamento, sendo possível explorar novos conhecimentos diretamente no processo produtivo, respondendo rapidamente às mudanças do ambiente. $\mathrm{O}$ conhecimento e as experiências adquiridas eram aplicadas ao longo dos anos de modo dinâmico, tanto na área tecnológica quanto de negócios, permitindo que a empresa se mantivesse na vanguarda tecnológica do segmento.

Diante do contexto apresentado, visualiza-se a teoria das capacidades dinâmicas como vantagem competitiva do período. Neste sentido, McKelvie e Davidson (2009) defendem a associação de capacidades dinâmicas com a exploração de oportunidades e geração de ideias. Os autores a conceituam como um feixe de outras capacidades como: de geração de ideias; introdução de rupturas de forma a criar dinamismo no mercado em que a empresa atua; desenvolvimento de novos produtos e serviços inovadores em quantidade de qualidade superior à 
concorrência e desenvolvimento de novos processos superiores aos concorrentes.

Identifica-se ainda neste período a capacidade absortiva, sendo um dos fatores componentes das capacidades dinâmicas (WANG; AHMED, 2007). Cohen e Levinthal (1990) destacam que a capacidade absortiva permite a empresa reconhecer o valor de uma nova informação externa, assimilá-la e aplicála para fins comerciais, então, é anterior e essencial para as capacidades de inovação da organização. Já no entendimento de Wang e Ahmed (2007), a capacidade absortiva é a habilidade da empresa em adquirir conhecimento externo, assimilá-lo com o interno e criar maneiras para aproveitar este novo saber.

\subsubsection{Período II - Crescimento e expansão do mercado (2006 - 2010)}

O empresário atribui grande mudança quando houve o crescimento e a expansão do mercado. Neste período a equipe de trabalho da empresa foi composta por 180 trabalhadores e a área fabril também foi ampliada.

$\mathrm{O}$ auge do negócio considerando este período deu-se devido a grande abertura de novos clientes, desenvolvendo produtos conforme a necessidade específica de cada cliente. A empresa para atender cada demanda específica de forma contínua adaptava-se, desenvolvendo-se concomitantemente, melhorando não só seus produtos, mas também os processos, estratégia e comportamento. A grande ampliação de clientes aconteceu porque estes cresceram mais que a sua capacidade de produção interna, necessitando assim de novos fornecedores e de terceirização de alguns componentes. Foi um grande desafio à gestão da empresa, pois o crescimento foi muito rápido.

Outro marco neste período foi a expansão para outros segmentos. Além do ramo agrícola ela passou a atender também os setores de armazenagem e pavimentação rodoviária. $\mathrm{O}$ acesso às linhas de crédito por parte da empresa e também dos clientes foi um fator significativo para tamanho crescimento e ampliação que passou a empresa.

Neste sentido, por introduzir na época novos produtos com grande tecnologia agregada e novas técnicas produtivas a empresa foi pioneira nos segmentos de atuação. Os clientes tinham a percepção da inovação, pois sua demanda e necessidade eram atendidas, otimizando seu processo produtivo.

Os produtos oferecidos e a tecnologia utilizada eram o grande diferencial competitivo, uma vez que não existia concorrência. Os processos de produção existentes na organização neste período eram desburocratizados, sendo um ponto bastante positivo, permitindo que a empresa se adaptasse com facilidade aos diferentes produtos e tecnologias, que despontavam no mercado. 
Em busca de constantes melhorias nos processos, a empresa desenvolvia também novas abordagens e modelos de gestão. Processos estes totalmente flexíveis, possibilitando rápidas mudanças no layout e nos sistemas. Se por algum determinado motivo a empresa não conseguia resolver um problema pelos métodos tradicionais, solicitava-se auxílio ao SENAI, diretamente com o fornecedor, assessoria e consultoria especializada.

Durante esta fase de crescimento e expansão do mercado a Metalúrgica Inovação esteve disposta e assumiu os riscos necessários para melhor explorar e aproveitar as oportunidades de crescimento. O principal limitador do período foi a escassez de mão de obra no mercado. Diante dessa fragilidade, a empresa optou por desenvolvê-la, formando internamente a aprendizagem operacional, onde ensinava os procedimentos técnicos necessários para a execução das tarefas e retêla, por meio de um programa de cargos e salários estendido a todos os trabalhadores.

Diante do exposto neste período de mudança da empresa percebe-se a presença da teoria das capacidades dinâmicas e a capacidade inovativa, como seu fator componente. Preponderante nesta perspectiva, Collis (1994) assevera que a capacidade dinâmica é a capacidade da organização em inovar de forma mais ágil e melhor que a concorrência.

A inovação, de modo geral, retrata um importante diferencial da competitividade e do desenvolvimento da organização estudada. A inovação organizacional, na visão de Wang e Ahmed (2004) é a capacidade de inovação global da organização, por meio da introdução de novos produtos para o mercado ou abertura de mercados, por meio do arranjo entre a orientação estratégica, o comportamento e o processo inovador.

O contexto da empresa permite ainda visualizar neste período a capacidade inovativa nas dimensões do produto, mercado, processo, comportamento e também do nível estratégico. Logo, as inovações são vitais para o sucesso do negócio (HAN; KIM; SRIVASTAVA, 1998).

\subsubsection{Período III - Crise e fusão (2011 - 2015)}

Neste evento a Metalúrgica Inovação teve um grande processo de mudança e precisou adaptar-se a uma nova e diferente realidade. Os negócios estavam andando muito bem, muitos contratos principalmente de produtos de pavimentação rodoviária com alto valor agregado. No entanto, em meados de 2011 instaura-se a crise política no país, tendo diretamente interferência e reflexos econômicos para a empresa.

Muitos dos clientes potenciais estão envolvidos em irregularidades públicas e a partir desta descoberta por parte do governo, instaura-se uma crise econômica e financeira na empresa, por meio do rompimento imediato dos contratos. No ato deste rompimento a maioria dos produtos 
solicitados já estavam acabados, sendo de custo altíssimo à Metalúrgica Inovação devido ao grande aporte tecnológico agregado. Sob esta perspectiva a empresa buscou alternativas, pois as finanças estavam comprometidas.

As alternativas não eram muitas após especulações e investigações no mercado. Para manter o negócio, a estrutura física e principalmente honrar o pagamento em dia dos salários do quadro de empregados, o empresário decidiu realizar a fusão com a empresa Stara. A Stara tem sua matriz na cidade de Não-Me-Toque/RS, sendo sua principal atividade a produção de máquinas agrícolas com tecnologia de ponta. Atua em todo o território nacional e esta presente nos cinco continentes, exportando seus produtos para mais de 35 países.

O processo de fusão veio para satisfazer ambas as organizações. Para a empresa estudada atendeu a necessidade de novos negócios com o aproveitamento da área física fabril e da mão de obra disponível e ociosa, como também pela busca do equilíbrio financeiro necessário para os ajustes econômicos que se impunham. Para a empresa Stara foi válido pela busca de ampliação do espaço fabril e pela emergência de contratação de mão de obra qualificada para o atendimento da sua produção. O processo de fusão suscitou novos negócios e solucionou falhas necessárias para o andamento dos mesmos.
Após um longo período de mudança e adaptação organizacional, a Metalúrgica Inovação passou a atuar na produção de produtos e componentes Stara S/A, de base agrícola.

Diante deste novo quadro, a empresa não teve como controlar as significativas mudanças a nível político e econômico. Assim, evidencia-se a presença da teoria das capacidades dinâmicas no período III e a capacidade adaptativa como seu fator componente preponderante.

Teece (2009) conceitua capacidades dinâmicas como a habilidade da organização de integrar, construir e reconfigurar suas competências em ambientes instáveis. Rindova e Kotha (2001) asseguram que as capacidades dinâmicas são refletidas por meio da capacidade de adaptação de uma organização, considerando essencialmente a flexibilidade estratégica dos recursos, o alinhamento interno dos recursos da empresa, a sua forma de organização e as necessidades permanentes de mudanças estratégias.

Nesta perspectiva, Burns e Stalker (1961) explicam que adaptação organizacional trata da interface entre ambiente e empresa, entendendo o ajuste entre o ambiente externo e a estrutura interna da organização. Wang e Ahmed (2004) asseveram que a capacidade adaptativa visa explicar como a empresa conecta as suas vantagens e recursos internos com as vantagens competitivas existentes no mercado. Esta capacidade torna a empresa 
capaz de capitalizar as emergentes oportunidades do mercado (MILES; SNOW, 1978; CHAKRAVARTHY, 1982; HOOLEY; LYNCH; JOBBER, 1992).

\section{CONSIDERAÇÕES FINAIS}

O estudo se propôs a analisar o processo de mudança estratégica pela concepção da teoria das capacidades dinâmicas na empresa Metalúrgica Inovação Ltda, numa perspectiva longitudinal, desde o inicio de suas atividades.

A pesquisa mostrou que a organização passou por três grandes etapas no negócio, caracterizando assim três fases distintas, interpretadas como períodos de mudança, ao longo dos últimos 15 anos.

Os resultados mostram que as mudanças da empresa foram conduzidas ora pela capacidade de identificar oportunidades do empresário, ora pela imposição do ambiente. As decorrências vão ao encontro da teoria das capacidades dinâmicas e de seus fatores componentes, sendo as capacidades absortiva, adaptativa e inovativa. Esta teoria apresentou-se adequada para explicar o contexto da organização, uma vez que trata-se de uma abordagem contemporânea que melhor elucida o enredo da empresa.

Deste estudo, conclui-se que o processo de mudança da Metalúrgica Inovação foi marcado pelo planejamento do empresário, em termos de concepção do negócio, crescimento, leitura de cenários, aproveitamento das oportunidades e também de adaptação do negócio às exigências e contingências da clientela e do mercado.

$\mathrm{O}$ processo de mudança de uma organização é complexo e envolve diversos fatores inter-relacionados e interdependentes. Diante disto, é possível afirmar que toda e qualquer organização irá passar ou já passou por um processo de mudança. Este processo será intenso ou não, dependendo das características da empresa e do ambiente no qual a mesma esta inserida.

Esta pesquisa oferece contribuições gerenciais, na medida em que possibilitou através da descrição e análise dos eventos de mudança, mostrar a relação da teoria com a prática, o que permite a reflexão e avaliação sobre o comportamento estratégico da organização. A teoria utilizada nesta pesquisa ofereceu aporte suficiente para relacionar os eventos internos e externos de relevância para contextualizar a atuação da Metalúrgica Inovação.

Por fim, este estudo limitou-se a contar a história da Metalúrgica Inovação com base na percepção do seu idealizador, ou seja, restringindo-se, portanto, à percepção e interpretação deste sujeito. Em termos de continuidade de estudos, sugere-se a realização de um novo estudo visando avaliar os novos rumos da organização após a fusão. Logo, considerando a visão de vários gestores. 


\section{REFERÊNCIAS BIBLIOGRÁFICAS}

ANDRADE, M. M. Introdução à metodologia do trabalho científico. 4 . ed. São Paulo: Atlas, 1999.

ANDREEVA, T.; CHAIKA, V. Dynamic

Capabilities: what they need to be dynamic?

St. Petersburg State University. São

Petersburgo. 2006.

AVLONITIS, G. J.; KOUREMENOS, A.; TZOKAS, N. Assessing the Innovativeness of Organizations and Its Antecedents: Project Innov. strat. European Journal of Marketing, vol. 28, n. 11, p. 5-28, 1994.

BURNS, T. A.; STALKER, G. The management of innovation. London:

Tavistock Publications, 1961.

CAMARGO, A. A. B.; MEIRELLES, D. S.

Capacidades Dinâmicas: o que são e como identificá-las? In: Encontro da Anpad, 2012, Rio de Janeiro. Rio de Janeiro: Anpad, 2012.

CHAKRAVARTHY, B. S. Adaptation: a promising metaphor for strategic management. Academy of Management Review, v. 1, p. 35-44, 1982.

CHILD, J.; SMITH, C. The context and process of organizational transformation: Cadbury Limited in its sector. Journal of Management Studies. p. 565-590, 1987.

COHEN, A. MBA: curso prático. Rio de Janeiro: Campus, 1999.

COHEN, M. D.; LEVINTHAL, D. A. Absorptive capacity: a new perspective on learning an innovation. Administrative Science Quarterly. n. 35, p. 128-152, 1990.

COLLIS, D. J. Research note: how valuable are organizational capabilities? Strategic Management Journal, p. 143-152, 1994.

CRESWELL, J. W. Projeto de pesquisa: métodos qualitativo, quantitativo e misto. 3. ed., Porto Alegre: Artmed, 2010.

DOSI, G.; NELSON, R. R.; WINTER, S. G. Introduction: the nature and dynamics of organizational capabilities. In: DOSI, G.; NELSON, R. R.; WINTER, S. G. The nature and dynamics of organizational capabilities. Oxford: Oxford Press, p. 1-22, 2000.

EISENHARDT, K. M.; MARTIN, A. Dynamic capabilities: what are they?

Strategic Management Journal, v. 21, n. 10-11, p. 1105-1121, 2000.

GERHARDT, T. E.; SILVEIRA, D. T. Métodos de Pesquisa. Universidade Federal do Rio Grande do Sul, 2009.

HAN, J. K.; KIM, N.; SRIVASTAVA, R. K. Market Orientantion and Organizational Performance: Is Innovation a Missing Link? Journal of Marketing. v. 62, p. 30-45, October, 1998.

HIGGINS, J. M. Innovate or evaporate: test \& improve your organization's iq: its innovation quotient. New Management, 1995.

HOOLEY, G. J.; LYNCH, J. E.; JOBBER, D. Generic marketing strategies. International Journal of Research in Marketing, v. 9, p. 75-89, 1992.

HREBINIAK, L.G.; JOYCE, W.F.

Organizational adaptation: strategic choice and environmental determinism.

Administrative Science Quarterly, v. 30, p. 336-349, 1985.

KIM, W. C.; MAUBORGNE, R. Strategy, value innovation, and the knowledge economy. Sloan Management Review, 40 (3), p. 41-53, 1999.

MARTINS, G. A. Manual para elaboração de monografias e dissertações. 2. ed. São Paulo: Atlas, 2000.

MCKELVIE, A.; DAVIDSON, P. From Resourse Base to Dynamic Capabilities: an investigation of New Firms. British Journal of Management, Oxford, n. 20, p. 63-80, 2009.

MILES, R.; SNOW, C. Organizational strategic, structure and process. New York: Mc Graw-Hill, 1978.

MINAYO, M. C. S. Pesquisa social: teoria, método e criatividade. Petrópolis: Vozes, 2008. 
MINTZBERG, H.; AHLSTRAND, B.; LAMPEL, J. Safári de estratégia. Porto Alegre:

Bookmann, 2010.

MINTZBERG, H. Patterns in strategy formation. Management Science, v. 24, n. 9, mai. p. 934-948, 1978.

NELSON, R. R. Why do firms differ, and how does it matter? Strategic Management Journal, v. 12, n. 8, p. 61-74, 1991.

NELSON, R. R.; WINTER, S. G. An Evolutionary Theory of Economic Change. Cambridge: Belknap Press, 1982.

PETTIGREW, A. M. Context and action in the transformation of the firm. Journal of Management Studies. p. 649-670, nov, 1987.

\section{PETTIGREW, A. M. Shapping Strategic}

Change. London: Sage, 1992.

PETTIGREW, A. M.; FERLIE, E.; McKEE, L. Shaping strategic change. London: Sage, p.1-30, 1992.

PRODANOV, C. C.; FREITAS, E. C. Metodologia do trabalho científico: métodos e Técnicas da pesquisa e do trabalho acadêmico. 2. ed. Novo Hamburgo: Feevale, 2013.

RINDOVA, V. P.; KOTHA, S. Continuous "morphing": competing through dynamic capabilities, form, and function. Academy of Management Journal, n. 44, p. 1263-1280, 2001.

SAUSEN, J. O. Gestão estratégica, competitividade e desenvolvimento: um olhar a partir das suas interrelações. In:

SIEDENBERG, D. R. (org.)

Desenvolvimento sob múltiplos olhares. Ijuí: Unijuí, 2012.

SMINIA, H. Process research in strategy formation: theory, methodology and relevance. International Journal of Management Reviews, v.2, n.1, p.97-125, 2009.
TALKE, K.; SALOMO, S.; KOCK, A. Top Management Team Diversity and Strategic Innovation Orientation: The Relationship and Consequences for Innovativeness and Performance. Journal of Product Innovation Management, v. 28, p. 819-832, 2011.

TEECE, D. J.; PISANO, G.; SHUEN, A. Dynamic capabilities and strategic management. Strategic Management Journal, v. 18, n. 7, p. 509-533, 1997.

TEECE, D. J. Dynamic capabilities \& strategic management. Oxford: Oxford University Press, 2009.

TRIVINÕS, A. N. S. Introdução à pesquisa em ciências sociais: a pesquisa qualitativa em educação. São Paulo: Atlas, 1987.

VASCONCELOS, F; CYRINO, A.

Vantagem competitiva: os modelos teóricos e a convergência entre estratégia e teoria organizacional. RAE - Revista de Administração de Empresas, out./dez. 2000, São Paulo: v.40, n.4, p.20-37.

WANG, C. L.; AHMED, P. K. Dynamic capabilities: A review and research agenda. International Journal of Management Reviews, v.9, n.1, p.31-51, 2007.

WHITTINGTON, R. The work of strategizing and organizing: for a practice perspective.

Strategic Organization, v.1, n.1, p.119-127, 2002.

WINTER, S. G. Economic 'Natural Selection' and the Theory of the Firm. Yale Economic Essays, v.4, p.225-272, 1964.

WINTER, S. G. Understanding Dynamic Capabilities. Strategic Management Journal, v.24, p.991-995, 2003.

YIN, R. K. Estudo de caso: planejamento e métodos. 3. ed. Porto Alegre: Bookman, 2005.

ZOLLO, M.; WINTER, S. G. Deliberate Learning and the Evolution of Dynamic Capabilities. Organization Science. v.13, n.3, p.339-351, Mai./Jun. 2002. 